\title{
Concept study on active shells driven by soft dielectric EAP
}

\author{
Patrick Lochmatter, Gabor Kovacs* \\ Laboratory for Materials and Engineering, Swiss Federal Laboratories for Materials Testing and \\ Research (Empa), Dubendorf, Switzerland
}

\begin{abstract}
Adaptive structures are capable to change their shape in a smart way in order to "adapt" to variable external conditions. Active shell structures with large out-of-plane deformation potential may be used to generate an interaction between the structural shape and the environment. Exemplarily, such shell-like actuators may be utilized for the propulsion of vehicles through air or water. Among the electroactive polymers (EAPs) especially soft dielectric EAP are promising for driving shell-like actuators due to their huge active strain potential and intrinsic compliancy.

The challenging task of this study was to explore the potential of the DE actuator technology for the design of shell-like actuators with the ability to perform complex out-of-plane deflections. We present and evaluate concepts for the design of active shell structures driven by soft dielectric EAP. Preliminary experiments were conducted for selected approaches in order to basically verify their principle of operation and to quantify their active out-of-plane deformation potential. These experiments showed that the so-called agonist-antagonist configuration, where pre-strained DE films are attached on both sides of a hinged backbone structure, holds good performance in terms of active out-of-plane deflections and forces.
\end{abstract}

Keywords: Electroactive polymers, soft dielectric EAP, dielectric elastomers actuators, shell-like actuator.

\section{INTRODUCTION}

Among the different classes of active materials, especially the soft dielectric EAP, a subgroup of the electroactive polymers (EAPs), are promising for muscle-like actuators ${ }^{1-4}$ due to their intrinsic compliancy and good overall performance. The combination of large active deformation, high energy density, good efficiency and prompt response is unique to dielectric elastomer (DE) actuators.

Soft dielectric EAP correspond to a compliant capacitor, where an elastomeric film is sandwiched between two compliant electrodes. Silicone and acrylic elastomers are most widely used as dielectric materials, due to their excellent mechanical (low elastic modulus of elasticity) and electrical (high dielectric constant and breakdown strength) properties $^{5,6}$.

When an electrical voltage is applied between the electrodes, the electrostatic forces (Maxwell stresses) from the charges on the electrodes squeeze the elastomer in thickness direction and the incompressible film expands in the plane. According to Pelrine et al. ${ }^{7}$ the equivalent pressure of the electrodes grows linearly with the dielectric constant of the film and quadratically with the applied electrical field. As soon as the voltage is switched off and the electrodes are short-circuited the film contracts back to its initial state.
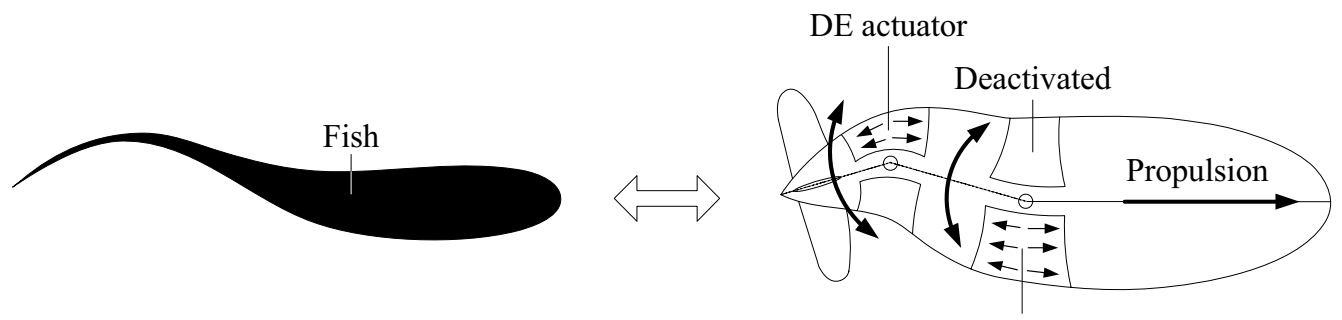

Activated

Figure 1: Inspired by the continuous shape changes of a swimming fish in water (left) a fish-like propulsion of a blimp in air, based on EAP, (right) was proposed ${ }^{8}$.

* mail: gabor.kovacs@empa.ch, phone: 0041 (0)1 82340 63, fax: 0041 (0)1 82340 11, internet: www.empa.ch 
Due to their unique properties, soft dielectric EAP are promising for active structures where large deformations are needed. In particular, active shells, which can accomplish quasi-continuous out-of-plane displacements, would meet a variety of applications, such as:

- Drag and/or oscillation reduction on wind-exposed structures (e.g. buildings, bridges, ropeway gondolas).

- Fish-like propulsion of objects through fluids (Fig. 1).

In general, active shells, here referred to as "shell-like actuators", which exhibit "organic shape changes" may be utilized to generate interactions between the surface of a structure and its environment.

In this concept study we explored different approaches for shell-like actuators driven by soft dielectric EAP. Introductorily, some preliminary considerations on shell-like actuators are discussed in section 2. In sections 3 and 4 promising concepts are presented and evaluated.

\section{PRELIMINARY CONSIDERATIONS}

\subsection{Requirements for the shell-like actuator}

The shell-like actuator based on soft dielectric EAP shall be a lightweight structure with (planar) dimensions in the macro-scale range and unbroken surfaces, which can actively exhibit large, quasi- or fully continuous out-of-plane deflections. In addition, the shell-like actuator has to be capable to withstand external loads acting upon its surface. Moreover, highly integrated structures with low mechanical complexity are preferred, which consist of commercially available components.

\subsection{Classification of the deformation potential}

According to the requirements, the shell-like actuator shall be capable to actively take complex out-of-plane shapes. In general, the deformation capabilities of such actuators may be classified into:

- Uniaxial deformation: The actuator actively performs deformations in one planar direction (only contraction or elongation; both elongation and contraction) or bend around one axis (only forth or back; both back and forth). As shown in figure 2, left, cylindrical shapes with variable radius of curvature result for instance from uniaxial bending deformations.

- Biaxial deformation: The actuator can actively deform in both planar directions (only contraction or expansion; both contraction and expansion) or bend around two perpendicular bending axes (only forth or back; both back and forth). When additionally both deformation types (planar deformation and/or bending) can be combined and their deformation amplitudes may be independently adjusted in both principal directions, a wide variety of shapes can be mapped (e.g. bowl or saddle shown in Fig. 2, center).

- Complex deformation: In the most general case, the actuator is capable to map irregular out-of-plane shapes. The range of mappable surface shapes is only limited by the (local) deformation potential of the actuator (Fig. 2, right).
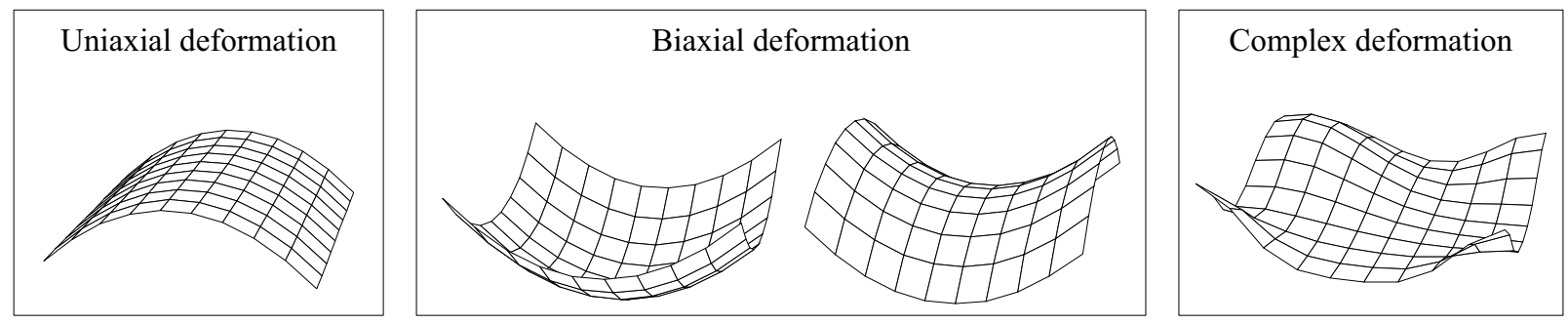

Figure 2: Different levels of active deformation potential of shell-like actuators.

\subsection{Inspiration by nature}

As an inspiration for shell-like structures, it is worthwhile to consider evolutionarily optimized solutions provided by nature. Batoid fishes (stingrays, skates, sawfishes and guitar fishes) for instance have wing-like bodies, which they use for propulsion (Fig. 3, left). During swimming, deflection waves travel along the wings. According to the number of waves moving across the wings, either undulatory, semi-oscillatory or oscillatory locomotion is performed". 
The wings of batoid fishes are mechanically supported by a skeletal system of serially arranged, radial bones that are connected via joints (Fig. 3, right). Muscles are spanned from the pectoral girdle to each radial bone segment. The outer skin layer encloses the system consisting of muscles and bones. The major tasks of the skeletal system are:

- Generating the needed structural stiffness to transfer the forces from the surrounding fluid as well as the internal muscle forces to the pectoral girdle.

- Maintaining the structural flexibility of the wing so that large deflections are possible.

With this configuration batoid fishes can perform quasi-continuous bending motions with their wings. Thereby, on the one hand the wing can bend around the body axis (flapping of the wings). On the other hand, however, the support between adjacent radials via the cartilaginous extensions couples the deflection motion of neighboring radials (e.g. for wave propagation along the wing).
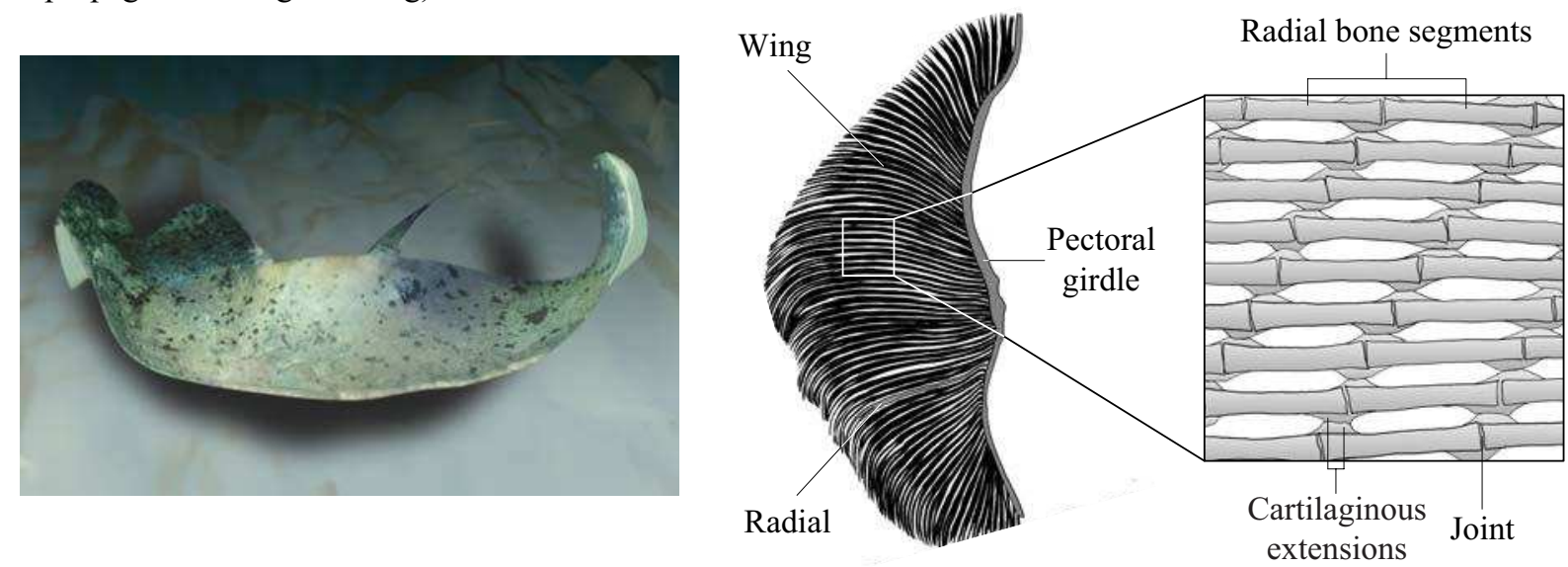

Figure 3: Batoid fishes (left) have a skeletal structure (right) consisting of radials, which are composed of serially arranged radial bone segments?

\subsection{Agonist-antagonist configurations}

Likewise, an active structure based on soft dielectric EAP requires high spatial resolution in active zones, which all need to be supplied/controlled independently, to achieve complex, quasi-continuous shape changes. Basically, out-of-plane deflections are induced by active generation of a gradient of in-plane stresses across the thickness of the shell-like actuator. With natural configurations, this is accomplished by muscles, which interact via a skeletal support structure.

\section{Conventional agonist-antagonist configuration}

Regarding the elastomers used as dielectric in DE actuators, in particular the commercially available acrylic film VHB 4910 from $3 \mathrm{M}$ has shown the best overall performances ${ }^{6}$. In order to reduce the activation voltage level of DE actuators from VHB 4910, the dielectric film needs to be strongly pre-strained in both planar directions. In analogy to the muscleskeletal structure proposed by nature, a configuration, where the pre-strained DE films as "artificial muscles" are attached to a flexible support structure, seems to be promising for the shell-like actuator. Thereby, the main requirements for the support structure are:

- Maintaining the DE film in its biaxially pre-strained state.

- Offer the mechanical degrees of freedom (DOF) needed for enabling the out-of-plane displacements of the actuator.

In contrast to the natural solution, where all muscles are attached to the pectoral girdle, we suggest independent segments each driven by a pair of artificial muscles. The proposed configuration is similar to the structure of a human arm, where two muscles are pre-strained via jointed bones (Fig. 4, left). With the active segment, two pre-strained DE films are mounted to a hinged support structure (Fig. 4, center). Under active expansion of each one of the pre-strained DE films, the support structure executes a rotational motion (Fig. 4, right). Thereby, the directions of motion of a human arm and the DE configuration are opposite to each other since biological muscles contract under activation, while soft dielectric EAP expand. 


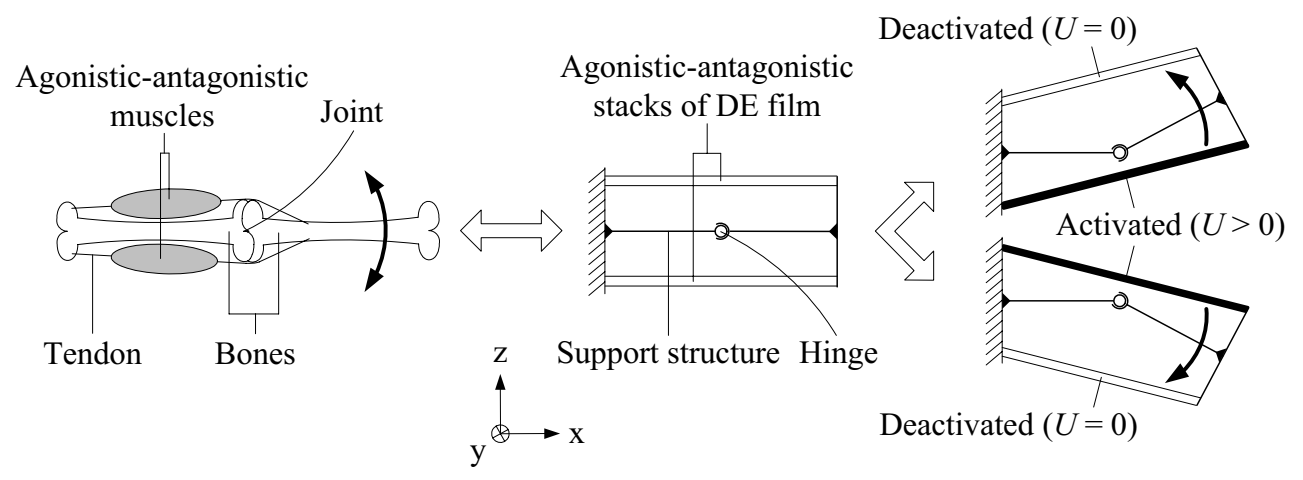

Figure 4: Biologically inspired agonist-antagonist configuration driven by soft dielectric EAP.

The angle of deflection achieved by the active segment can be optimized by variation of the segment geometry (aspect ratio and position of the hinge joint) and the setup of the DE actuators (pre-stretch ratios). The actively generated deflection force, on the other hand, can be scaled by mounting stacked DE film layers to the support structure.

\section{Enhanced agonist-antagonist configuration}

Obviously, no active motion results from the conventional agonist-antagonist configuration when equally activating both $\mathrm{DE}$ actuators. In this case, the tensile loads in the $\mathrm{DE}$ films are simply reduced by the transmission of the equivalent electrode pressure into planar directions.

By replacing the hinged support structure with an elastic core (e.g. springs) a force equilibrium is taken in the freestanding state between the two pre-strained stacks of DE films and the compressed core (Fig. 5, left). Under activation of only the upper or lower DE actuator, the corresponding films expand and a coupled extensional/rotational deformation of the enhanced segment arises (Fig. 5, center). In addition, the enhanced segment elongates in $x$ direction, when both DE actuators are simultaneously activated (Fig. 5, right).

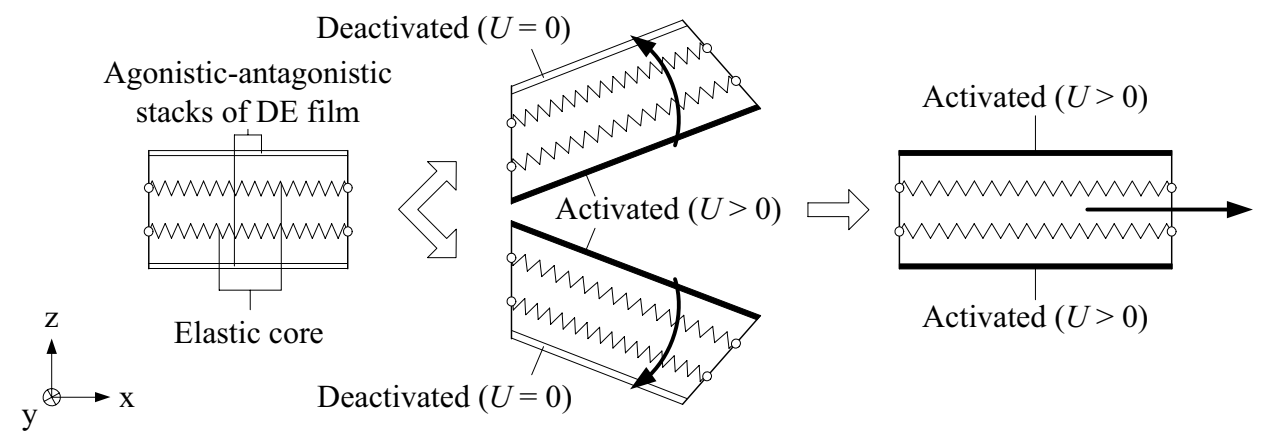

Figure 5: Enhanced agonist-antagonist configuration.

\section{Inflated agonist-antagonist configuration}

So far, the pre-strain of the DE actuators was preserved by a mechanical core consisting of either a hinged structure or a compressed, elastic core. In order to avoid any mechanical core structure and thus primarily reduce the weight of the system, the coated dielectric films may be pre-strained by compressed gas.

A potential approach for an inflated agonist-antagonist configuration is presented in figure 6 . The segment consists of rigid side faces, to which DE film stacks are attached on the top and bottom. By inflation of the segment with gas the DE films are pre-strained since the films bulge out and the side faces move apart. Note that the gas used for inflation may be any lighter-than-air gas (e.g. helium) if further reduction in weight is required. Under activation of the upper or lower DE actuator, the corresponding films expand and a extensional/rotational deformation of the inflated segment arises. When simultaneously activating both DE actuators, an elongation in $x$ direction is expected. 


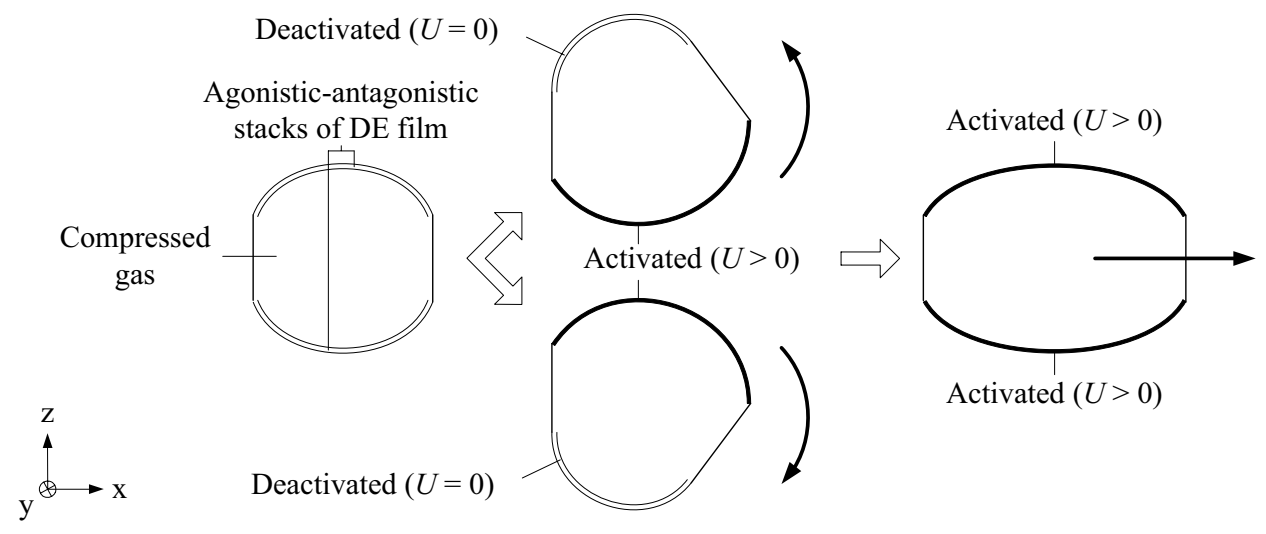

Figure 6: Inflated agonist-antagonist configuration.

\section{PRESENTATION OF THE CONCEPTS}

In this section the conceptual approaches for the shell-like actuators based on soft dielectric EAP are introduced and corresponding preliminary experimental results are presented. The concepts were classified according to their structure and deformation potential respectively into the following groups:

\section{- Approaches based on the agonist-antagonist configuration: $\quad$ (A) Conventional agonist-antagonist configuration \\ (B) Enhanced agonist-antagonist configuration \\ (C) Inflated agonist-antagonist configuration \\ - Other configurations: \\ (D) Film stack configuration \\ (E) Active framework configuration}

\subsection{Approaches based on the agonist-antagonist configuration}

(A) Conventional agonist-antagonist configuration

For shell-like actuators based on the conventional agonist-antagonist configuration, which are capable to execute uniaxial bending deflections, the support structure of each segment requires one rotary DOF. The goal is to find a flexible and lightweight support structure (backbone), which offers marginal friction since rather low forces are expected from the DE films. For the experimental characterization, uniaxial bending actuators consisting of several interconnected active segments were implemented. Three basic types of support structures were taken into account

(i) Rolling elements: The required flexibility of the support structure may be achieved by curved elements, which roll on each other. The pre-strained DE films press these elements against each other. While this core provides low friction the rolling elements may shift relative to each other. In addition, they may loose their contact under large external tensile loads. We experimentally implemented an arrangement of six lines consisting each of four connected polymer spheres $(\varnothing=40 \mathrm{~mm})$ (Fig. 7, left). In order to prevent the spheres from loosing their contact, the actuator was suspended from two long threads fixed at both ends of the actuator.

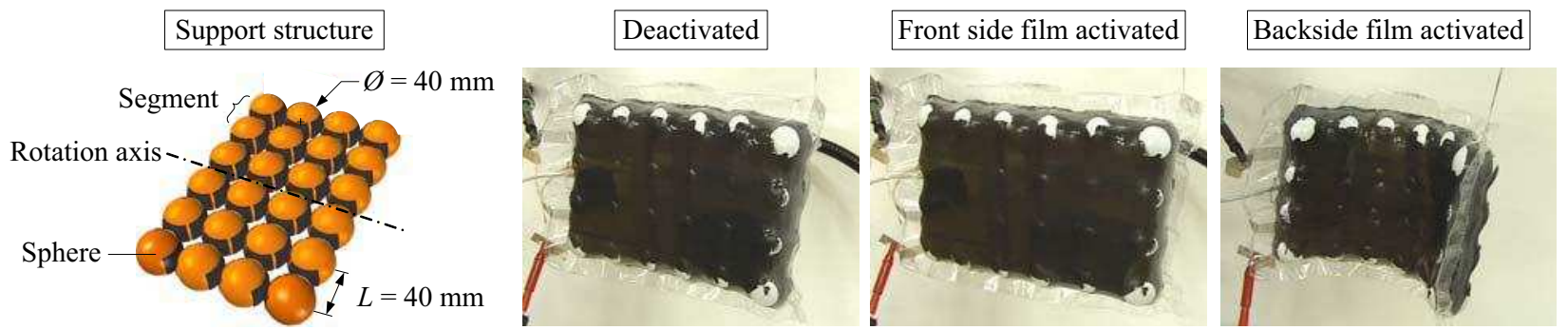

Figure 7: Experimental implementation of a uniaxial bending actuator based on a core structure composed of elements rolling on each other. 
(ii) Supported elements: When the contact between the support structure elements is established via pins, which are supported in sockets, the lateral shift is prevented, while the rotational flexibility of the core is maintained (Fig. 8, left). Nevertheless, the parts of the support structure still loose their contact, when large external tensile loads act in longitudinal direction. For experimental implementation, five reinforced cylindrical PVC profiles (type 556090 from Fennel, length $200 \mathrm{~mm}, \varnothing=32 \mathrm{~mm}$ ) with spherical end pieces were connected via each two parallel, tapered metal pins (length $44 \mathrm{~mm}$ ), which were seated in conical sockets of metal.
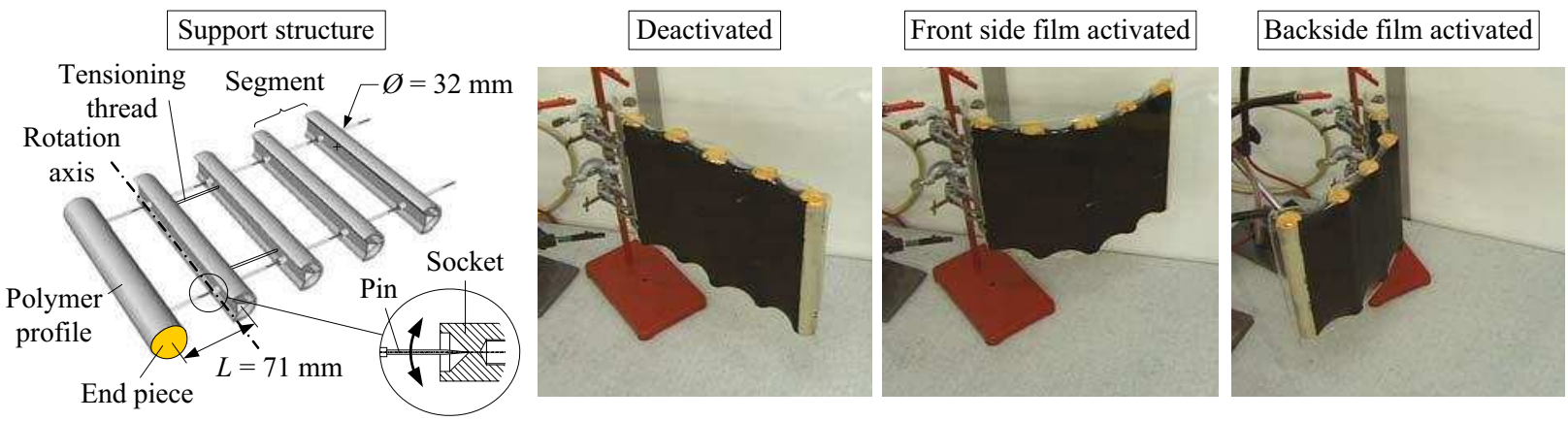

Figure 8: Experimental implementation of a uniaxial bending actuator based on a core structure composed of supported elements.

(iii) Hinged elements: Only when the core parts are fully hinged, external loads do not affect the proper operation of the actuator (Fig. 9, left). However, increased friction has to be accepted. A hinged core composed of five lines consisting each of three connected polymer spheres $(\varnothing=40 \mathrm{~mm})$ was implemented. The polymer struts (length $48 \mathrm{~mm}$ ), which were fixed at their one end and hinged at the other end, connected each line of spheres.
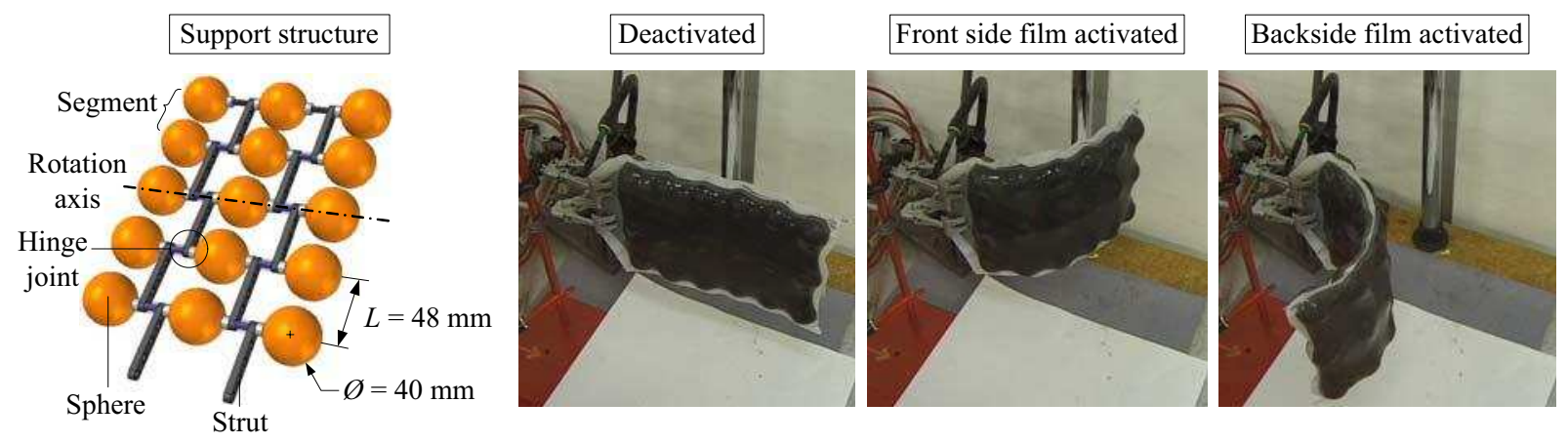

Figure 9: Experimental implementation of a uniaxial bending actuator based on a core structure composed of hinged elements.

For all shell-like actuators the acrylic film VHB 4910 (3M) was utilized. The films were symmetrically pre-strained (pre-stretch ratios $\lambda_{x}{ }^{(i)} \times \lambda_{y}{ }^{(i)}=4 \times 4$ ) and mounted on rigid frames. The compliant electrodes, which consisted of a mixture of graphite (type TIMREX SLP 30 from TIMCAL) and silicone oil (type DC 200/50cs Fluid from Dow Corning), were applied by brush-coating.

During assembly of the actuator, the support structure was sandwiched between the two framed and coated DE films. After sticking together the film layers all around the support structure, the actuator was cautiously cut out of the frame. For the testing procedure, the actuators were oriented upright in order to prevent the influence of gravity on the active displacement.

Similar deflection results were achieved with all three selected support structures (Fig. 7-9). Under electrical activation of each one of the DE films with DC high voltages of up to $4 \mathrm{kV}$, the actuators displayed strong uniaxial bending deflections back and forth (Fig. 7-9, right). Typically, a fast initial rotational motion up to large angles of deflection was observed immediately after activation. This motion was followed by a slow creep deformation governed by the viscous properties of acrylic VHB $4910(3 \mathrm{M})$. As soon as the DE film was short-circuited, the actuators quickly returned towards their original shape. 


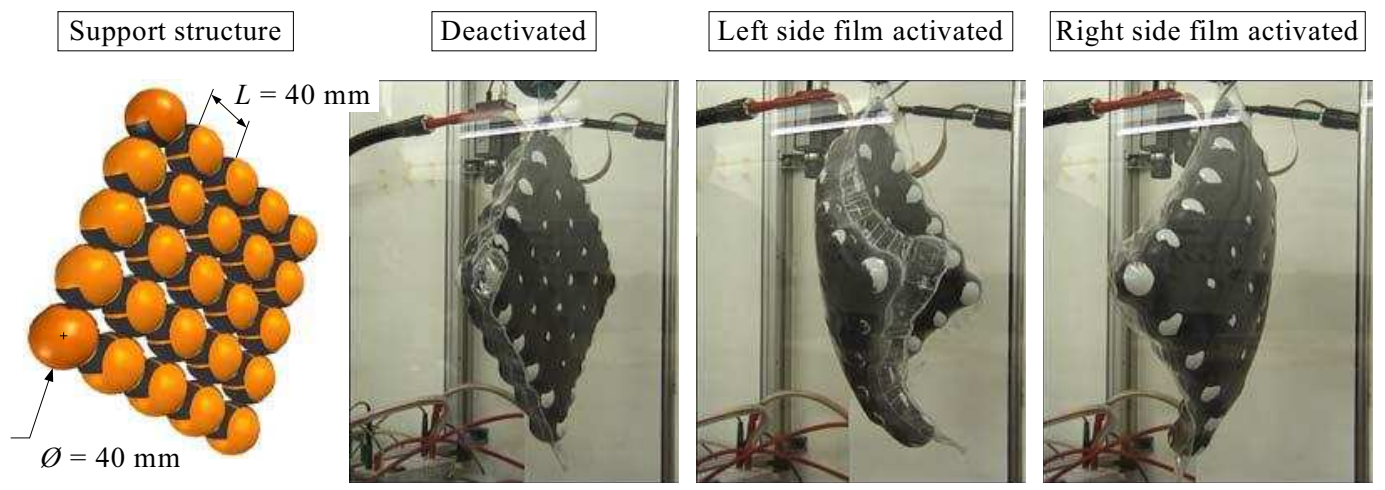

Figure 10: Experimental implementation of a biaxial bending actuator based on the conventional agonist-antagonist configuration.

In order to adapt the conventional agonist-antagonist configuration to a shell-like actuator, which can exhibit biaxial bending deformations, an appropriate support structure is required. The support structure has to preserve the biaxial prestrain in the DE film, while mechanically allowing for a biaxial bending deformation. According to theoretical investigations, the core structure must allow for shear distortions to admit biaxial bending (a sheet of paper e.g. can not properly biaxially bend due to its intrinsic shear stiffness). When the core structure allows for shear distortions, however, the resulting shell-like actuator would provide no shear stiffness and would thus collapse under in-plane loads. Despite the theoretical prediction, we implemented a biaxial bending actuator based on the conventional agonistantagonist configuration. The core consisted of an array of closely fitting polymer spheres, which were sandwiched between two pre-strained (pre-stretch ratios $\lambda_{x}^{(i)} \times \lambda_{y}^{(i)}=4 \times 4$ ) and coated films of VHB 4910 (3M) (Fig. 10, left). Under activation of the DE films with DC high voltages of $4 \mathrm{kV}$ fast biaxial bending deflections with large amplitudes were observed in both directions (Fig. 10, right).

According to the theoretical investigation, however, the spheres in the core did not simply roll off each other during biaxial deformation of the actuator, they additionally had to slide relative to each other. This effect could be verified, since the initially planar actuator displayed a residual curvature after several activation cycles.

\section{(B) Enhanced agonist-antagonist configuration}

A uniaxial bending/elongation segment based on the enhanced agonist-antagonist configuration was experimentally implemented and characterized in terms of its free strain deformation potential. The support structure consisted of two long polymer beams (dimensions $320 \times 10 \times 40 \mathrm{~mm}$ ) with five intermediary pieces of elastic foam (each of size $45 \times 40 \times$ $30 \mathrm{~mm}$ ) (Fig. 11, left).

During manufacturing of the segment, the elastic foam core was strongly compressed before the pre-strained (prestretch ratios $\lambda_{x}{ }^{(i)} \times \lambda_{y}{ }^{(i)}=4 \times 4$ ) and brush-coated (mixture of graphite powder (type TIMREX SLP 30 from TIMCAL) and silicone oil (type DC 200/50cs Fluid from Dow Corning)) films of VHB 4910 (3M) were mounted on both sides of the core.

After removal of the external clamping, the compressed foam core expanded against the pre-strained DE films. In the free-standing equilibrium state, the DE films were asymmetrically pre-strained with pre-stretch ratios of about $\lambda_{x}^{(i)} \times \lambda_{y}^{(i)} \approx 7 \times 4$ (Fig. 11, center).

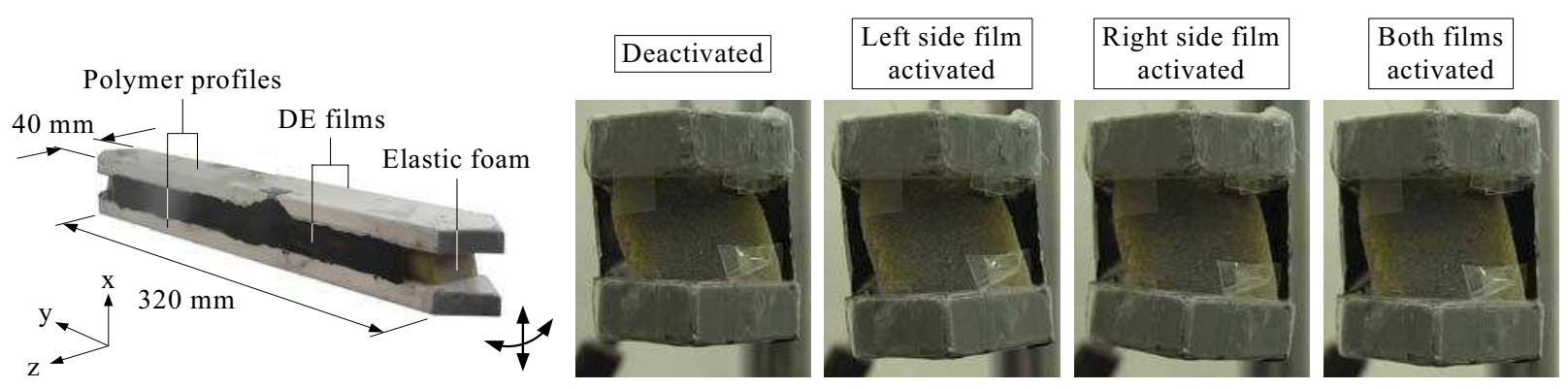

Figure 11: Experimental implementation of a uniaxial bending/elongation segment based on the enhanced agonist-antagonist configuration. 
For the experimental characterization, the enhanced segment was oriented horizontally. The upper polymer profile was clamped to an external support, while the lower one remained free to execute the active motions. Under short-time activation of each one of the DE films with DC voltages of $4 \mathrm{kV}$ angles of deflection of about \pm 6 degrees around the $y$ axis were observed (Fig 11, right). Under simultaneous activation of both DE films elongations of up to $20 \%$ resulted in $x$ direction (Fig. 11, rightmost).

While the principle of operation of the enhanced agonist-antagonist segment could be verified, in particular the amplitudes in active deflection were fairly low. According to modeling results, the active deflections can be increased by reducing the bending stiffness of the core (using e.g. thinner foam slab). Obviously, the major issue with the enhanced agonist-antagonist segment is to find an elastic element, which provides low bending stiffness and does not bulge out under in-plane compression by the pre-strain loads of the DE film layers.

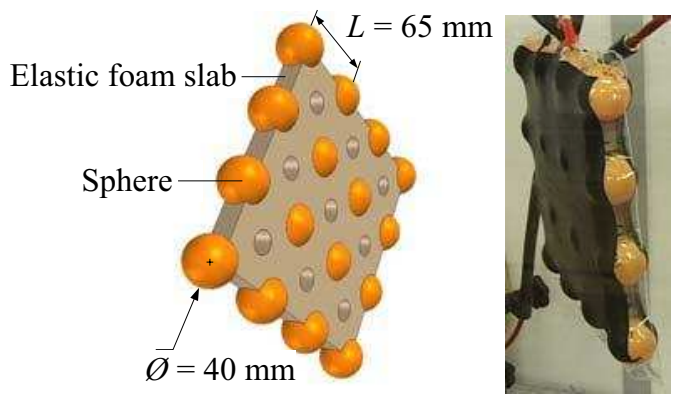

Figure 12: Experimental implementation of a biaxial bending/elongation actuator based on the enhanced agonist-antagonist configuration.

By adapting the enhanced agonist-antagonist configuration to a two-dimensional arrangement, a shell-like actuator with biaxial bending potential is accomplished since the elastic core allows for shear deformations. A core consisting of a slab of elastic foam (size $200 \times 200 \times 30 \mathrm{~mm}$ ) was selected for the experimental implementation. Polymer spheres $(\varnothing=40 \mathrm{~mm})$ were embedded for attaching the pre-strained DE films (Fig. 12, left).

In contrast to the uniaxial bending/elongation segment, which was compressed before applying the pre-strained DE films, in this case the DE films were over-strained before applying them to the relaxed core. After cutting the actuator out of the frame, the contracting DE films compressed the elastic core. However, only little compression of the core resulted, when only one DE film layer was on both sides of the core (Fig. 12, right). As a consequence, only little deformation amplitudes were achieved under activation with up to $4 \mathrm{kV}$. Nevertheless, the actuator displayed biaxial expansion in planar directions under activation of the DE films on both sides, while biaxial bending deflections were observed under activation of solely one of the DE films. In order to reach larger active deformations, several stacked DE film layers may be mounted to the elastic core, which would thus be compressed stronger.

\section{(C) Inflated agonist-antagonist configuration}

Based on the inflated agonist-antagonist configuration, a shell-like actuator was implemented, which can uniaxially bend. Two film layers of VHB $4910(3 \mathrm{M})$ were pre-strained (pre-stretch ratios $\lambda_{x}{ }^{(i)} \times \lambda_{y}{ }^{(i)}=4 \times 4$ ) and brush-coated with electrodes of a mixture of graphite (type TIMREX SLP 30 from TIMCAL) and silicone oil (type DC 200/50cs Fluid from Dow Corning). The pre-strained and coated films were mounted to a structural core consisting of six adjoining pairs of linked polymer spheres $(\varnothing=40 \mathrm{~mm})$. The resulting actuator was inflated with air (Fig. 13, left).
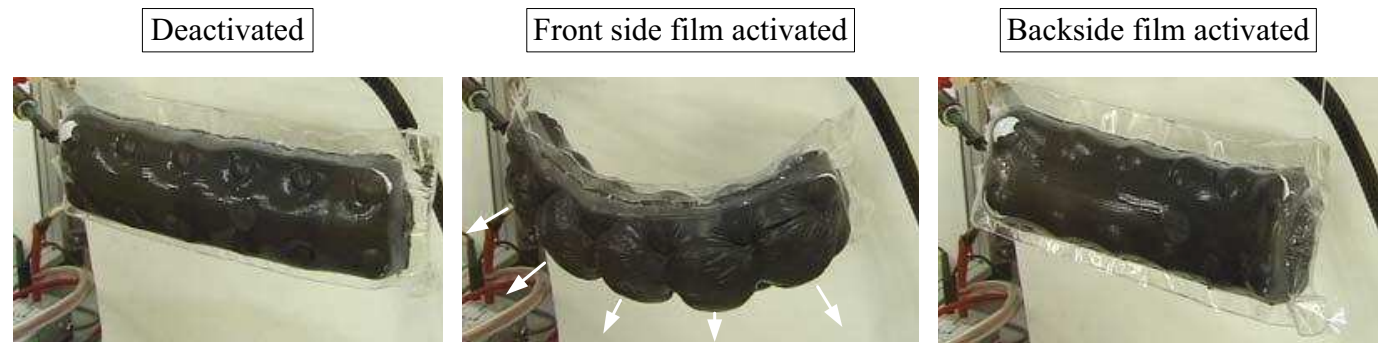

Figure 13: Experimental implementation of a uniaxial bending actuator based on the inflated agonist-antagonist configuration. 
For testing, the actuator was suspended from two long threads attached to its ends. Under activation of each one of the DE films the actuator exhibited strong uniaxial bending deflections back and forth (Fig. 13, right). As illustrated in figure 13, center, the DE film strongly bulged out under activation due to the inner gas pressure.

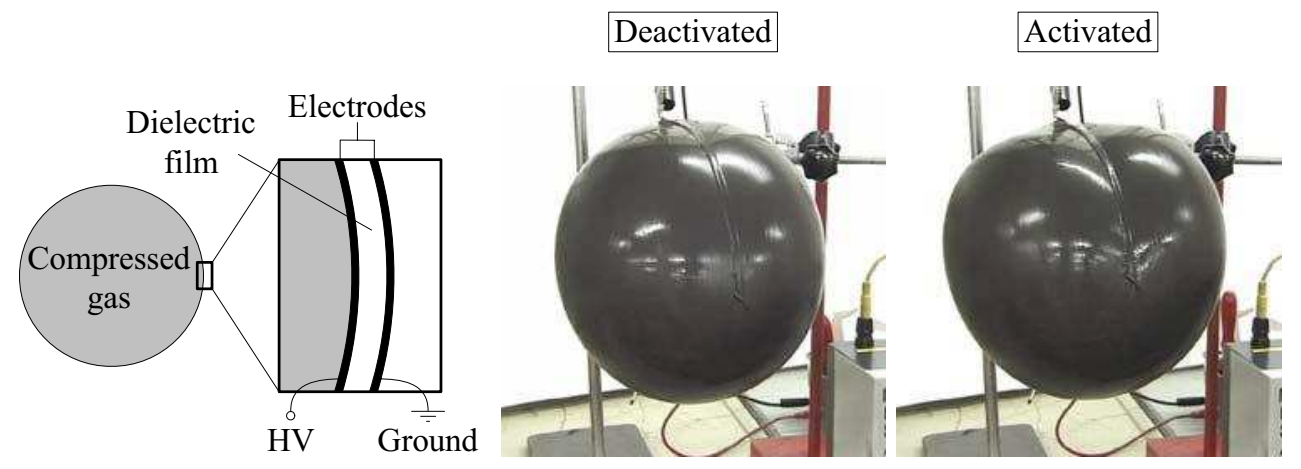

Figure 14: Inflated balloon based on soft dielectric EAP, which expands in volume under activation.

Obviously, an inflated elastic membrane tends to take a spherical shape as known from balloons. Thus, we additionally tried to implement a balloon actuator based on soft dielectric EAP (Fig. 14, left). A folded bag from VHB 4910 (3M) was filled with loose graphite powder (type TIMREX SLP 30 from TIMCAL). The elastic bag was then inflated with air until the dielectric film reached the desired pre-strain level (balloon diameter about $25 \mathrm{~cm}$ ). By shaking the inflated balloon the loose graphite powder was distributed across the inner surface of the sticky film. The outer electrode, which consisted of a mixture of graphite powder (type TIMREX SLP 30 from TIMCAL) and silicone oil (type DC 200/50cs Fluid from Dow Corning) was painted with a brush on the outside surface of the balloon.

As expected, the DE balloon actuator expanded considerably in volume under activation with voltages of up to $5 \mathrm{kV}$ (Fig. 14, right). However, the pre-strained film VHB 4910 (3M) was not fully gastight so that the balloon needed to be pumped up once in a while.

By interconnecting many (small) DE balloon actuators in a bilayer arrangement a shell-like actuator results, which can exhibit biaxial bending deformations (Fig. 15, left). Under activation of the upper or the lower balloon layer a biaxially curved out-of-plane deflection is expected (Fig. 15, center). When activating both balloon layers the actuator elongates in both planar directions (Fig. 15, right). This configuration, however, has not yet been experimentally implemented.

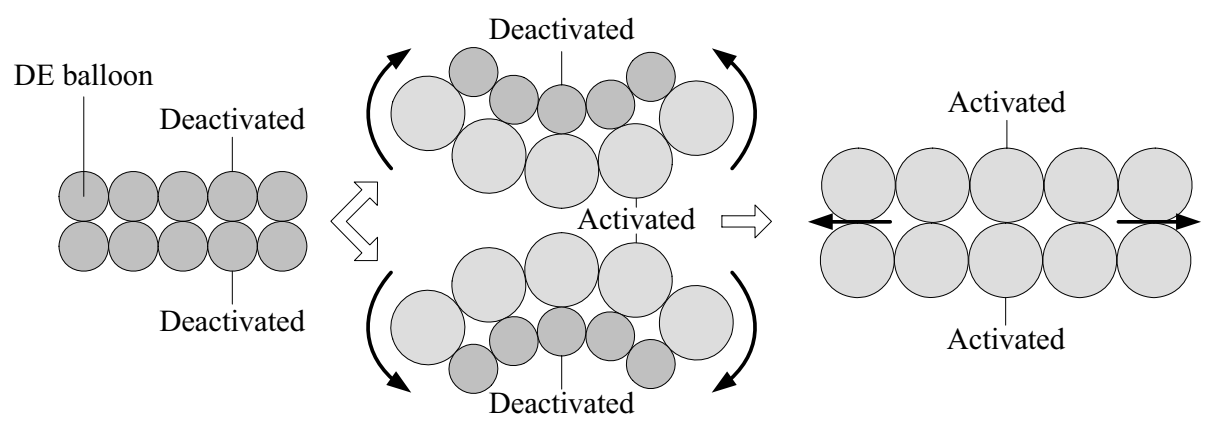

Figure 15: Shell-like actuator consisting of a bilayer arrangement of balloon actuators based on soft dielectric EAP.

\subsection{Other configurations}

(D) Film stack configuration

In contrast to the supported configurations, out-of-plane deflections may be achieved by continuously constraining the active expansion of the DE films under activation as well. Following the suggestion by Pelrine et al. ${ }^{7}$ continuous bending deformations may be achieved with a bimorph actuator. While this actuator bends under activation of each one DE film layer, it elongates in the plane when both layers are simultaneously activated. 
Unlike the agonist-antagonist configurations, the DE films of stack configurations are not pre-strained. By activation of all DE film layers of a stack with equal activation voltage an expansion in both planar directions results (Fig. 16, left). When the activation voltage increases across the film layers, however, a biaxial bending deformation is expected. By mechanically constraining the DE film stack in one direction (e.g. by applying fibers) uniaxial elongation results under equal activation of all layers and uniaxial bending arises when increasing the voltage level across the film layers.

When arranging unconstrained and constrained stack segments in a checker pattern, as e.g. shown in figure. 16, right, the resulting shell-like actuator may accomplish specific out-of-plane deflections. Obviously, the actuator expands in both planar directions under uniform activation of all layers. Whereas biaxial bending (bowl) is displayed when applying an increasing voltage level across the film layers. Selective elongation in one planar direction is expected, when the constrained segments of every second line of the pattern are activated. Moreover, selective uniaxial bending (cylindrical) is obtained, when these segments are activated with increasing voltage level across the film layers.
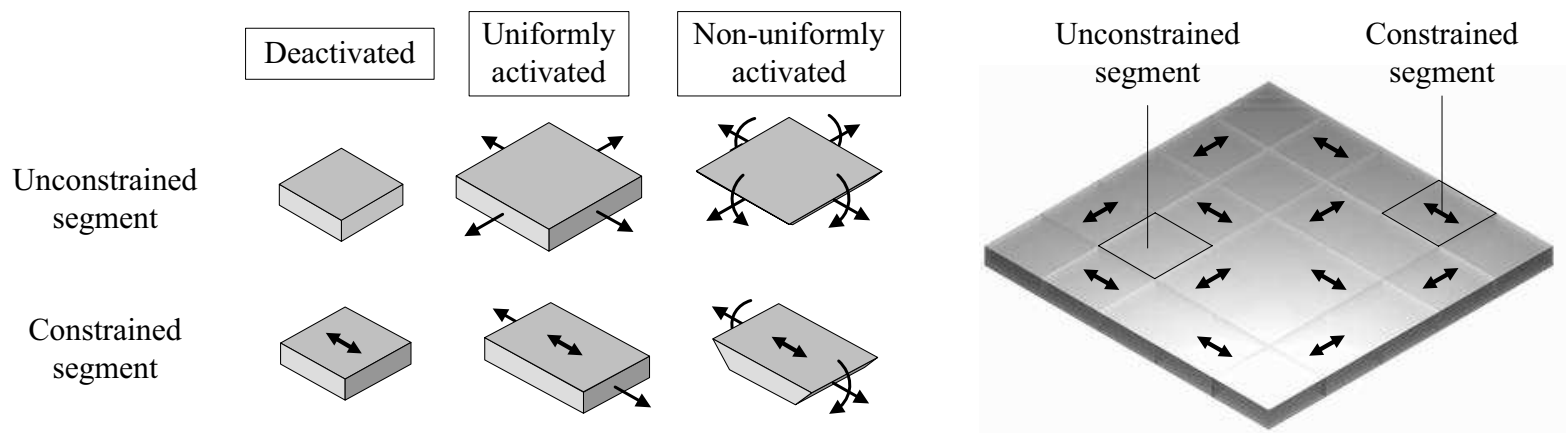

Figure 16: Shell-like actuator based on segments of stacked DE film layers.

In order to experimentally investigate the basic deformation potential of stacked DE film actuators mainly bilayer and multilayer configurations were taken into account:

- Bilayer configuration: The bilayer actuator consisted of two (not pre-strained) stacked dielectric films (VHB F9473PC from $3 \mathrm{M}$, size $110 \times 110 \mathrm{~mm}$ ), which were mounted to a non-compliant intermediate electrode (conductive tape 9713 from 3M, size $100 \times 100 \mathrm{~mm}$ ) (Fig. 17, left). The compliant outer electrodes consisted of a mixture of silicone oil (type DC 200/50cs Fluid from Dow Corning) with graphite powder (type TIMREX SLP 30 from TIMCAL) and were manually painted with a brush.

Due to the very low intrinsic stiffness of the stack, the actuator was suspended from several long threads. Beneficially, the active bending deformations were not obstructed by this support. Under electrical activation of each single DE layer, a strong uniaxial bending deformation back and forth was observed (Fig. 17, right). The activation voltage could be increased up to $8.5 \mathrm{kV}$, where the bending curvature of the actuator resulted in a closed loop.

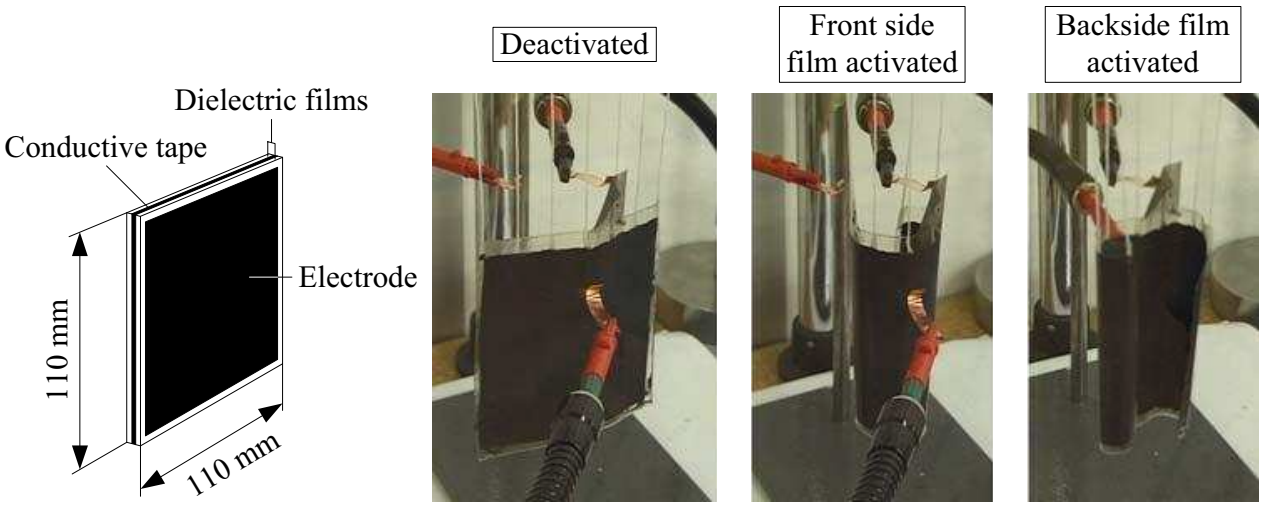

Figure 17: Experimental implementation of a uniaxial bending actuator based on a bilayer film stack configuration. 
- Multilayer configuration: The multilayer actuator was composed of twenty layers of (not pre-strained) dielectric film (VHB F9473PC from 3M, size $125 \times 125 \mathrm{~mm}$ ). The electrodes consisted of a mixture of silicone (RTV23/A7 from Swiss-Composite) and carbon black (Ketjenblack EC-300J from Akzo Nobel) and were manually painted. The first and the second ten film layers were connected each to one high voltage feeding line (HV 1 and HV 2, Fig. 18, left). Since the actuator provided sufficient intrinsic bending stiffness it was simply clamped at one corner for testing.

When activating only one part of the DE film layers of the stack (DC high voltage at either HV 1 or HV 2) with voltages of up to $5.75 \mathrm{kV}$ mainly uniaxial bending deformations back and forth around the vertical axis were observed (Fig. 18, center). Obviously, the bending deformation around the horizontal axis was small due to the dominant influence of gravity in comparison to the electroactive forces. Under electrical activation of all DE film layers the stack actuator expanded distinctively in planar directions (Fig. 18, right).

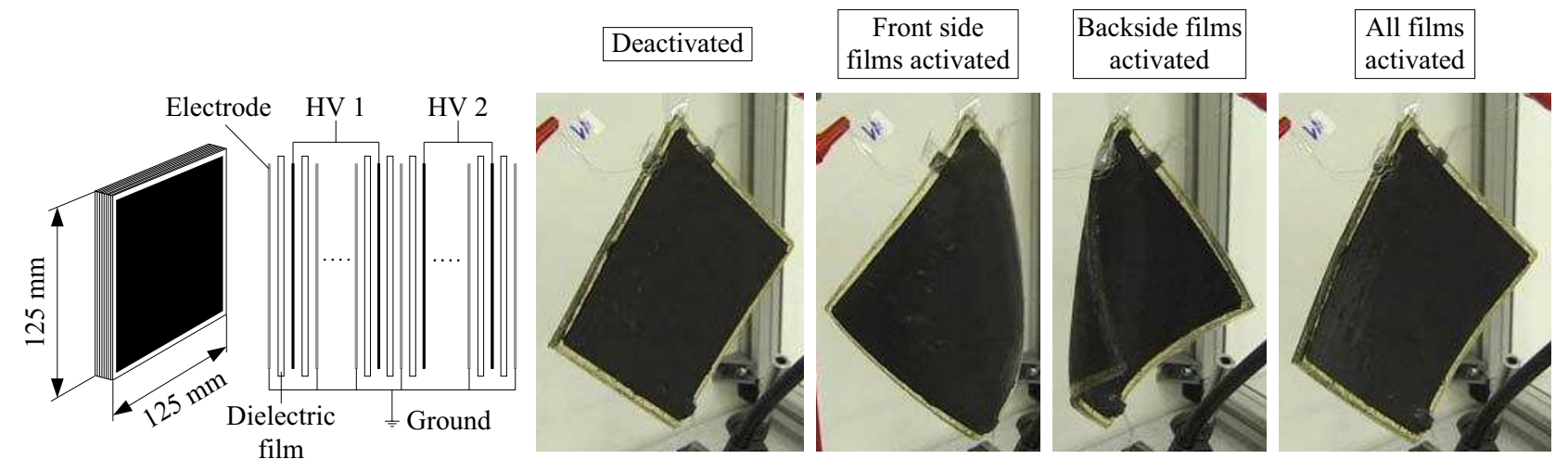

Figure 18: Experimental implementation of a biaxial bending/elongation actuator based on a multilayer film stack configuration.

\section{(E) Active framework configuration}

In order to map a predetermined surface shape, one may use an active framework structure, whose struts can actively change their length. Mazzone ${ }^{10}$ suggested a bilayer framework structure denoted as "Smart-Mesh" consisting of interconnected telescopic struts for haptic feedback applications. By (so far manually) changing the length of the rods the statically determinate framework can change its shape and map even hanging over shapes. Based on simulations the huge deformation potential of this active structure was verified.
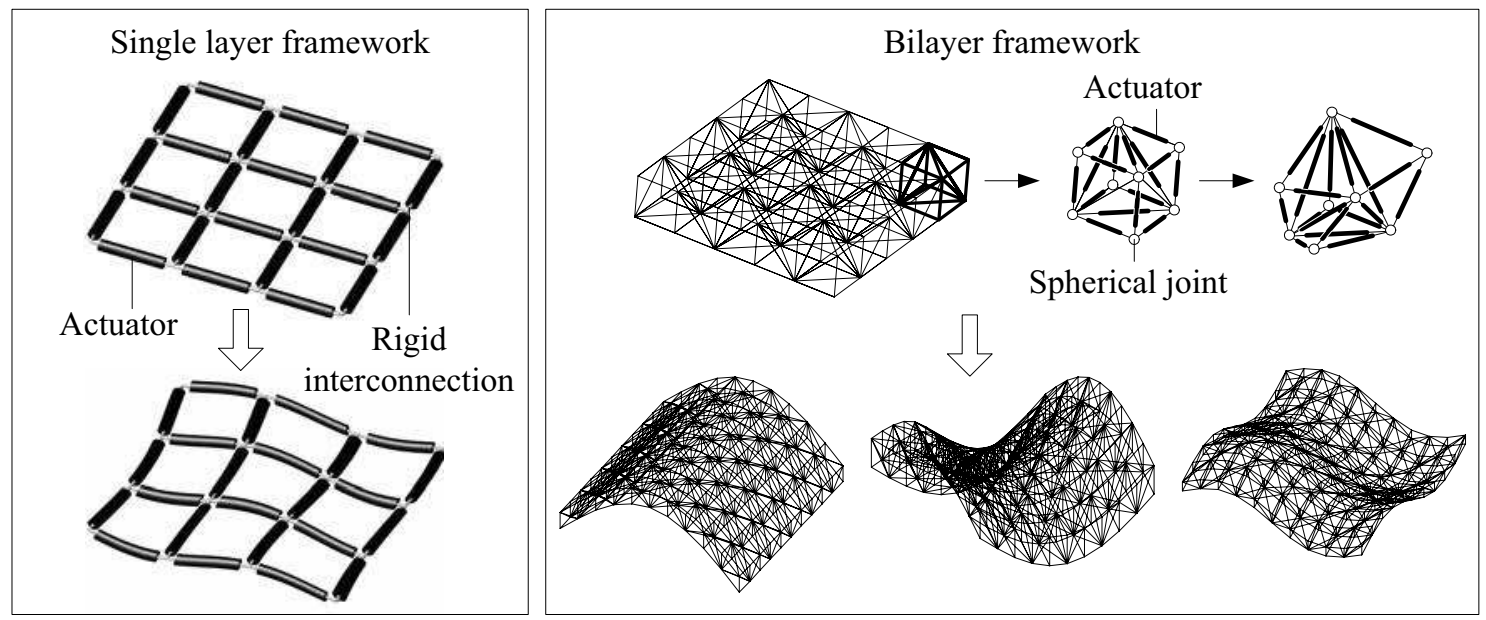

Figure 19: Single layer or bilayer active framework configuration 
As potential candidates for shell-like actuators, single layered and bilayered framework structures were taken into account:

- Single layer framework: Pei et al. ${ }^{11}$ have demonstrated rolled actuators based on soft dielectric EAP, which can execute specific bending/elongation deformations in different directions under activation. By rigidly interconnecting such actuators at their ends an active mesh is established, which can map complex out-of-plane shapes (Fig. 19, left).

- Bilayer framework: The bilayer framework consists of linear actuators, which are interconnected at their ends via spherical joints (Fig. 19, right). Under specific elongation of the actuators the shape of the framework structure can be varied. In particular, rolled DE actuators, which execute linear elongations under activation ${ }^{12}$ seem appropriate for this application. Since the struts of the active framework may also be compressively loaded, the rolled actuators need to be enhanced by a mechanical core guidance (e.g. by a telescope) in order to prevent buckling.

This configuration, however, was not completed and thus no results are available for quantitative evaluation yet.

\section{EVALUATION OF THE CONCEPTS}

While the conventional agonist-antagonist configuration, (A), shows large active displacements, the complexity and weight of the support structure remains detrimental. The enhanced configuration, (B), offers more deformation modes, including proper biaxial bending. This approach, however, showed only little active deformation in the preliminary experiments. The strength of approach (C) is its lightweight structure. However, the dielectric film needs to be completely gastight. Moreover, the active skin tends to bulge out under activation due to the inner gas pressure and thus the resulting out-of-plane deflections and forces of such actuators are expected to be rather low.

According to the evaluation, the actuator consisting of not pre-strained stacked DE films, (D), complies with most criteria. In particular, this is the only approach, where a continuous curvature of the surface is achieved under activation. While DE film layers are at a certain distance with the agonist-antagonist configurations, (A)-(C), they are directly stacked on each other in configuration (D). As a result, stacked film actuators offer less bending stiffness and therefore only low active out-of-plane forces.

Active frameworks, (E), offer a wide range of structural designs and active deflections. However, the frameworks need to be covered by a soft membrane to achieve an unbroken surface as required for the shell-like actuator. Moreover, the benefit from soft dielectric EAP as active membrane is not incorporated in this approach. This means that the framework rods, as simple linear actuators, could be realized based on conventional actuator technologies as well (e.g. hydraulic, pneumatic cylinders).

In table 1 an assessment of the presented concepts is given according to the criteria for the shell-like actuator introduced in section 2 .

\begin{tabular}{|c|c|c|c|c|c|}
\hline & (A) & (B) & (C) & (D) & (E) \\
\hline Degree of complexity of deformation & ○ & $\bigcirc$ & $\bigcirc$ & $\bigcirc$ & $\bigcirc$ \\
\hline $\begin{array}{l}\text { Amplitude of active deformation } \\
\text { (according to estimation or preliminary experiments) }\end{array}$ & $\bigcirc$ & 0 & ○ & $\bigcirc$ & $\bigcirc$ \\
\hline $\begin{array}{l}\text { Amplitude of active forces } \\
\text { (according to estimation) }\end{array}$ & $\bigcirc$ & ○ & 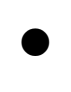 & 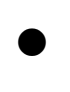 & $\bigcirc$ \\
\hline Continuity of actuator surface & $\bigcirc$ & $\bigcirc$ & $\bigcirc$ & $\bigcirc$ & \\
\hline Scalability of spatial deformation resolution & ○ & $\odot$ & ○ & $\bigcirc$ & 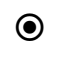 \\
\hline Lowness of mechanical complexity & 0 & $\odot$ & O & $\bigcirc$ & \\
\hline Lightweightness & ○ & $\odot$ & $\bigcirc$ & $\bigcirc$ & \\
\hline Simplicity for manufacturing & ○ & ○ & ○ & $\bigcirc$ & 0 \\
\hline
\end{tabular}

Table 1: Assessment of the conceptual approaches for the shell-like actuator, symbols: $\bigcirc=$ good, $\odot=$ satisfactory, $\bullet=$ bad. 
Obviously, all approaches have their benefits and drawbacks. Nevertheless, especially the conventional agonistantagonist configuration seems to hold promising potential for its application to shell-like actuators, which are able to exhibit uniaxial bending deformations. Thereby, the support structure allows for pre-straining of the DE films, which leads to reduced activation voltages. Moreover, the range of deformation of the shell-like actuator is controlled by the mechanical flexibility of the support structure. Finally, a tradeoff between the actively achievable displacements and forces can be found by proper design of the support structure.

\section{CONCLUSIONS}

In this study we considered a wide variety of variants for shell-like actuators based on soft dielectric EAP, which can actively execute out-of-plane deflections.

Based on so-called agonist-antagonist configurations promising actuators were experimentally implemented. Especially the uniaxial bending actuator with a hinged support structure showed large active deflections in both directions. Moreover, this configuration allows a simple tuning of the active performance in terms of active deflections and forces by proper selection of the distance between the active film layers. For near DE film layers large angles of deflection are expected, while with distant layers large active forces may be generated.

The conceptual approach of stacking many not pre-strained DE film layers on each other shows nice deflection potential. The low intrinsic stiffness of these actuators, however, is one major drawback.

The active frameworks hold large potential for active shape changes. However, with this concept the soft dielectric EAP can not introduce its unique properties as compliant active skin.

In our future work we will focus on the improvement of the shell-like actuators based on the agonist-antagonist configuration, which exhibit uniaxial bending deflections. On the one hand, the support structure will be optimized to achieve a lightweight structure with low inner friction. On the other hand, the DE actuators will be tuned by examining the effect of different pre-strain setups and the scalability of the active forces by applying several stacked DE film layers.

\section{REFERENCES}

1. Ashley S., “Artificial Muscles”, Scientific American, 2003, p 52-59.

2. Pelrine R., R. Kornbluh, Q. Pei, S. Stanford, S. Oh and J. Eckerle, "Dielectric Elastomer Artificial Muscle Actuators: Toward Biomimetic Motion" Proc. SPIE in Smart Struct. and Mat.: Electroactive Polymer Actuators and Devices, San Diego (USA), vol. 4695, p 126-37, 2002.

3. Bar-Cohen Y., "Electroactive Polymer (EAP) Actuators as Artificial Muscles - Reality, Potential and Challenges" (Ed: Y. Bar-Cohen), SPIE, Bellingham, 2001.

4. Kornbluh R., "Dielectric elastomer artificial muscle for actuation, sensing, generation, and intelligent structures", Mat. Techn., 19(4), p 216-24, 2004.

5. Carpi F., A. Mazzoldi and D. De Rossi, "High-strain dielectric elastomer for actuation", Proc. SPIE in Smart Struct. and Mat.: Electroactive Polymer Actuators and Devices, San Diego (USA), vol. 5051, p 419-22, 2003.

6. Pelrine R., R. Kornbluh, Q. Pei and J. Joseph, "High-speed electrically actuated elastomers with strain greater than 100\%" Science, 2000, 287(5454), p 836-839.

7. Pelrine R. E., R. D. Kornbluh and J. P. Joseph, "Electrostriction of polymer dielectrics with compliant electrodes as a means of actuation", Sensors and Actuators A: Physical, 64(1), p 77-85, 1998.

8. Michel S., G. Kovacs and P. Lochmatter, "Antrieb für einen Leichter-als-Luft-Flugapparat", Swiss Patent No. 00660/05, 2005.

9. Schaefer J.T. and A.P. Summers, "Batoid wing skeletal structure: Novel morphologies, mechanical implications, and phylogenetic patterns", Journal of Morphology, 2005, 264(3), p 298-313.

10. Mazzone A., "Deformable Mechanical Structure for Physical Generation of Objects and Provision of Wide Area Haptic Feedback", PhD thesis, Swiss Federal Institute of Technology (ETH), Zurich, Switzerland, 2004.

11. Pei Q., M. Rosenthal, S. Stanford, H. Prahlad and R. Pelrine, "Multiple-degrees-of-freedom electroelastomer roll actuators", Smart Materials and Structures, 2004, 13(5), p N86-N92.

12. Pei Q., R. Pelrine, S. Stanford, R. Kornbluh and M. Rosenthal, "Electroelastomer rolls and their application for biomimetic walking robots", Synthetic Metals, 2003, 135-136, p 129-131. 